\title{
EXTREMAL PROPERTIES OF WEAKLY STARLIKE $p$-VALENT FUNCTIONS
}

BY

JAMES A. HUMMEL( $\left.{ }^{1}\right)$

In a paper appearing elsewhere, the writer defined the class of weakly starlike $p$-valent functions [3]. This class was defined in terms of functions whose arguments were "increasing on the boundary", but it was shown that the property given in Definition 1 below also characterized this class. For our purposes here, it is enough to use the property in Definition 1.

Definition 1. A function $f(z)$ will be said to be in the class $W_{p}$ if and only if $f(z)$ is regular in the unit disc $U=\{z|| z \mid<1\}$, has exactly $p$ zeros there at points $z_{1}, z_{2}, \ldots, z_{p}$ (some may be multiple), and

$$
f(z)=[h(z)]^{p} \prod_{i=1}^{p} \Psi\left(z, z_{i}\right),
$$

where $h(z)=z+b_{2} z^{2}+b_{3} z^{3}+\cdots$ is a normalized univalent starlike function in $U$ and for each $i$,

$$
\Psi\left(z, z_{i}\right)=\left(z-z_{i}\right)\left(1-z_{i}^{*} z\right) / z .
$$

(The asterisk indicates the complex conjugate.)

It should be observed that $\Psi(z, 0) \equiv 1$. This class properly contains the class of $p$-valent starlike functions considered by earlier writers such as Robertson [4] and Goodman [2]. This observation was already made by Bender, who introduced what was essentially this same class of functions and considered some of the extremal properties [1]. One difference here is in the normalization. The classes considered in the papers mentioned above were normalized by demanding that the first nonvanishing coefficient of $f(z)$ be 1 . Here, we demand that it be the product of the negatives of the nonzero zeros of $f(z)$.

Since the normalization is just a matter of multiplying by a constant, it is not very important. However, to see why we choose the normalization given here, observe the function

$$
\begin{aligned}
k\left(z, z_{1}\right)= & \frac{z}{(1+z)^{2}} \Psi\left(z, z_{1}\right)=a_{0}+a_{1} z+\cdots+a_{n} z^{n}+\cdots \\
= & -z_{1}+\left(1+2 z_{1}+\left|z_{1}\right|^{2}\right) z-\left(2+3 z_{1}+z_{1}^{*}+2\left|z_{1}\right|^{2}\right) z^{2} \\
& +\cdots+(-1)^{n+1}\left[n+(n+1) z_{1}+(n-1) z_{1}^{*}+n\left|z_{1}\right|^{2}\right] z^{n}+\cdots
\end{aligned}
$$

Received by the editors December 2, 1966.

(1) Work on this paper was supported by a grant from the National Science Foundation, NSF GP-3897, while the writer was on sabbatical leave at Imperial College, University of London. 
which is in the class $W_{1}$. Here, for all $n \geqq 1$,

$$
\left|a_{n}\right| \leqq n\left(1+\left|z_{1}\right|\right)^{2} \text {. }
$$

However, if we normalized by dividing through by $-z_{1}$ so as to have the first coefficient 1 , then the best bound for the coefficient of $z^{n}$ would be

$$
n\left(1 /\left|z_{1}\right|+2+\left|z_{1}\right|\right) \text {. }
$$

This bound has the unfortunate characteristic of tending toward infinity as $z_{1} \rightarrow 0$.

It is the purpose of this paper to discuss some of the extremal properties of functions in the class $W_{p}$. Some of these were obtained first by Goodman [2]. He was considering a different class of functions, but his results apply in this case also. Further results were obtained by Bender [1]. Here, we merely make some observations concerning these earlier results for the sake of completeness. The main new results of this paper concern the lower bounds for $\operatorname{Re}\left\{z f^{\prime} \mid f\right\}$ for functions of this class. Since it was shown in [1] that this lower bound must go to zero as $|z| \rightarrow 1$, the actual nature of this bound is of some interest.

Definition 2. For any $f(z) \in W_{p}$, let $z_{1}, z_{2}, \ldots, z_{p}$ be the zeros of $f(z)$, let $r_{i}=\left|z_{i}\right|$ for each $i$, let $R_{M}=\max \left\{r_{i} \mid i=1,2, \ldots, p\right\}, R_{m}=\min \left\{r_{i} \mid r_{i} \neq 0, i=1, \ldots, p\right\}$, and let $r=|z|$.

Now, the coefficients of a function $h(z)$ in (1) are dominated by the coefficients of $z /(1-z)^{2}$. The coefficients of $\Psi\left(z, z_{i}\right)$ in (2) are dominated by the coefficients of $\Psi\left(z,-r_{i}\right)=(1 / z)\left(z+r_{i}\right)\left(1+r_{i} z\right)$. This domination is preserved in the product since all of the coefficients are real and positive. We have the following theorem, essentially due to Goodman [2]. (See also Bender [1].)

THEOREM 1. Let $f(z)=a_{0}+a_{1} z+a_{2} z^{2}+\cdots \in W_{p}$. Then $\left|a_{n}\right|$ is less than or equal to the coefficient of $z^{n}$ in

$$
F_{M}(z)=\frac{1}{(1-z)^{2 p}} \prod_{i=1}^{p}\left(z+r_{i}\right)\left(1+r_{i} z\right) .
$$

This is less than or equal to the coefficient of $z^{n}$ in

$$
F_{1}(z)=\left(z+R_{M}\right)^{p}\left(1+R_{M} z\right)^{p} /(1-z)^{2 p}
$$

which is, in turn, less than the coefficient of $z^{n}$ in

$$
F_{0}(z)=(1+z)^{2 p} /(1-z)^{2 p} \text {. }
$$

The first two bounds are sharp, while no better bound can be obtained than the third which does not involve the moduli of the zeros of $f(z)$.

The function $\Psi\left(z, z_{i}\right)$ maps $|z|=r$ onto an ellipse whose foci are at $w=\left(1 \pm r_{i}\right)^{2}$. The maximum modulus occurs at one vertex, and, since the origin is on the major axis and not between the foci, the minimum modulus occurs at the other vertex. Using the well-known bounds for the modulus of $h(z)$, it is then easy to verify all of the following bounds, most of which is again due to Goodman [2] (see also Bender [1]). 
THeOREM 2. Let $f(z) \in W_{p}$. Then for any $z \in U$,

$$
\frac{1}{(1+r)^{2 p}} \prod_{i=1}^{p}\left|z-z_{i}\right| \cdot\left|1-z_{i}^{*} z\right| \leqq|f(z)| \leqq \frac{1}{(1-r)^{2 p}} \prod_{i=1}^{p}\left|z-z_{i}\right| \cdot\left|1-z_{i}^{*} z\right|,
$$

$$
\begin{aligned}
\frac{1}{(1+r)^{2 p}} \prod_{i=1}^{p}\left|r-r_{i}\right| \cdot\left(1-r_{i} r\right) & \leqq|f(z)| \leqq \frac{1}{(1-r)^{2 p}} \prod_{i=1}^{p}\left(r+r_{i}\right)\left(1+r_{i} r\right), \\
\frac{\left|r-R_{0}\right|^{p}\left(1-R_{0} r\right)^{p}}{(1+r)^{2 p}} & \leqq|f(z)| \leqq \frac{\left(r+R_{M}\right)^{p}\left(1+R_{M} r\right)^{p}}{(1-r)^{2 p}}
\end{aligned}
$$

where

and

$$
\begin{aligned}
R_{0} & =R_{m} & & \text { if } 0 \leqq r<R_{m} \\
& =r & & \text { if } R_{m} \leqq r \leqq R_{M} \\
& =R_{M} & & \text { if } R_{M}<r<1,
\end{aligned}
$$

$$
|f(z)| \leqq(1+r)^{2 p} /(1-r)^{2 p}
$$

All except the last are sharp. No better bound than (9) can be obtained which does not involve the moduli of the zeros of $f(z)$.

Notice that the bounds here are progressively wider. In (6), one has the sharpest bounds, but at the cost of knowing $z$ and all of the $z_{i}$. In (7), all of the moduli of the $z_{i}$ are assumed known (and only the modulus of $z$ ). For (8), only the moduli of the largest and smallest of the nonzero $z_{i}$ need be assumed. Finally, (9) is independent of the $z_{i}$ entirely. This last result is itself a good justification for the normalization chosen in Definition 1.

If $f(z)=\sum a_{n} z^{n}$ and $g(z)=\sum c_{n} z^{n}$ where all the $c_{n}$ are real with $\left|a_{n}\right| \leqq c_{n}$, then for any $z,\left|f^{\prime}(z)\right| \leqq g^{\prime}(r)$. This observation together with Theorem 1 gives the proof of the following results. The inequality (10) is essentially that given by Bender [1].

THEOREM 3. Let $f(z) \in W_{p}$. Then for any $z \in U$

$$
\begin{aligned}
& \left|f^{\prime}(z)\right| \leqq F_{m}^{\prime}(r) \\
& \left|f^{\prime}(z)\right| \leqq F_{1}^{\prime}(r)=p \frac{\left(1+R_{M}\right)^{2}(1+r)\left(r+R_{M}\right)^{p-1}\left(1+R_{M} r\right)^{p-1}}{(1-r)^{2 p+1}}, \\
& \left|f^{\prime}(z)\right| \leqq F_{0}^{\prime}(r)=4 p \frac{(1+r)^{2 p-1}}{(1-r)^{2 p+1}}
\end{aligned}
$$

Inequalities (10) and (11) are sharp and (12) is best possible in the same sense as Theorems 1 and 2.

Finding bounds for $\operatorname{Re}\left\{z f^{\prime} \mid f\right\}$ is more difficult. Upper bounds are relatively easy to find, but the more interesting lower bounds are troublesome. For any $f(z) \in W_{p}$ we have

$$
\frac{z f^{\prime}(z)}{f(z)}=p \frac{z h^{\prime}(z)}{h(z)}+\sum_{i=1}^{p} \frac{z \Psi^{\prime}\left(z, z_{i}\right)}{\Psi\left(z, z_{i}\right)}
$$


Bounds for the first term are well known. It is the remaining terms which cause the difficulty.

It is easily verified that

where

$$
\frac{z \Psi^{\prime}\left(z, z_{i}\right)}{\Psi\left(z, z_{i}\right)}=\frac{z_{i}-z_{i}^{*} z^{2}}{\left(z-z_{i}\right)\left(1-z_{i}^{*} z\right)}=\frac{r_{i}}{1-r_{i}^{2}}\left[\eta-\frac{1}{\eta}\right]
$$

$$
\eta=\left(z-z_{i}\right) /\left(r_{i} z-z_{i} / r_{i}\right) .
$$

This transformation maps $|z|<1$ onto $|\eta|<1$. The circle $|z|=r$ is mapped to the circle

$$
C_{r}: \eta=\delta+R \cos \phi+i R \sin \phi, \quad 0 \leqq \phi \leqq 2 \pi,
$$

where

$$
\delta=r_{i}\left(1-r^{2}\right) /\left(1-r_{i}^{2} r^{2}\right), \quad R=r\left(1-r_{i}^{2}\right) /\left(1-r_{i}^{2} r^{2}\right) .
$$

Let $\rho=|\eta|$. Then for any point on $C_{r}$

$$
\rho^{2}=\delta^{2}+R^{2}+2 R \delta \cos \phi,
$$

from which we can deduce

$$
\operatorname{Re}\{\eta\}=\delta+R \cos \phi=\left(\rho^{2}+\delta^{2}-R^{2}\right) / 2 \delta .
$$

Now let $|z|=r$. Observe that $\operatorname{Re}\{1 / \eta\}=\operatorname{Re}\left\{\eta^{*} /|\eta|^{2}\right\}=\operatorname{Re}\left\{\eta / \rho^{2}\right\}$. We find

$$
\begin{aligned}
\operatorname{Re}\left\{\frac{z \Psi^{\prime}\left(z, z_{i}\right)}{\Psi\left(z, z_{i}\right)}\right\} & =\frac{r_{i}}{\left(1-r_{i}^{2}\right)} \operatorname{Re}\left\{\eta-\frac{1}{\eta}\right\} \\
& =\frac{r_{i}}{\left(1-r_{i}^{2}\right)} \operatorname{Re}\left\{\eta-\frac{\eta}{\rho^{2}}\right\} \\
& =\frac{r_{i}}{\left(1-r_{i}^{2}\right)}\left[1-\frac{1}{\rho^{2}}\right] \operatorname{Re}\{\eta\} \\
& =\frac{r_{i}}{\left(1-r_{i}^{2}\right)}\left[1-\frac{1}{\rho^{2}}\right] \frac{\left[\rho^{2}+\delta^{2}-R^{2}\right]}{2 \delta} \\
& =\frac{\left(1-r_{i}^{2} r^{2}\right)}{2\left(1-r_{i}^{2}\right)\left(1-r^{2}\right)}\left[\rho^{2}-1-\left(R^{2}-\delta^{2}\right)+\frac{\left(R^{2}-\delta^{2}\right)}{\rho^{2}}\right] .
\end{aligned}
$$

The circle $|\eta|=\rho$ intersects the circle $C_{r}$ in two conjugate points and therefore $\rho$ suffices to determine the value of the above expression. To find the maximum or minimum of $\operatorname{Re}\left\{z \Psi^{\prime} / \Psi\right\}$ it suffices to find the maximum of

$$
V\left(\rho^{2}\right)=\left[\rho^{2}-1-\left(R^{2}-\delta^{2}\right)+\left(R^{2}-\delta^{2}\right) / \rho^{2}\right]
$$

for a fixed $R$ and $\delta$ as $\rho^{2}$ varies between the limits $(R-\delta)^{2}$ and $(R+\delta)^{2}$.

If $r<r_{i}$, then $V\left(\rho^{2}\right)$ is negative and strictly increasing in the range under consideration. The maximum and minimum values occur at $\rho^{2}=(R+\delta)^{2}$ and $(R-\delta)^{2}$ respectively, giving the results listed in Lemma 1 for $r<r_{i}$. These are sharp. 
If $r>r_{i}$, then $R^{2}-\delta^{2}>0$ and $V\left(\rho^{2}\right)$ decreases from $+\infty$ when $\rho^{2}=0^{+}$to 0 at $\rho^{2}=R^{2}-\delta^{2}$ and on down to its minimum which occurs at $\rho^{2}=\left(R^{2}-\delta^{2}\right)^{1 / 2}$. It then increases monotonically, passing through 0 when $\rho^{2}=1$.

Since $(R-\delta)^{2}<R^{2}-\delta^{2}$, the maximum value of $V\left(\rho^{2}\right)$ is positive, occurring when $\rho^{2}=(R-\delta)^{2}$. This gives the result for $M\left(r, r_{i}\right)$ stated in Lemma 1 below for the case $r>r_{i}$. This is sharp.

The minimum of $V\left(\rho^{2}\right)$ occurs at $\rho^{2}=\left[R^{2}-\delta^{2}\right]^{1 / 2}$ or at $\rho^{2}=(R+\delta)^{2}$, whichever is smaller. Set

$$
u(r)=\frac{R^{2}-\delta^{2}}{(R+\delta)^{4}}=\frac{\left(r-r_{i}\right)}{\left(1-r_{i} r\right)} \frac{\left(1+r_{i} r\right)^{3}}{\left(r+r_{i}\right)^{3}} .
$$

This function is zero when $r=r_{i}$, is positive for $r_{i}<r<1$ and equals 1 at $r=1$. If, for a given $r, u(r)<1$ then the minimum of $V\left(\rho^{2}\right)$ occurs at $\rho^{2}=\left[R^{2}-\delta^{2}\right]^{1 / 2}$, while if $u(r)>1$ the minimum occurs at $(R+\delta)^{2}$.

We find

$$
u^{\prime}(r)=\frac{r_{i}\left(1-r_{i}^{2}\right)\left(1+r_{i} r\right)^{2}}{\left(r+r_{i}\right)^{4}\left(1-r_{i} r\right)^{2}}\left[r^{2}-\left(\frac{1+r_{i}^{2}}{2 r_{i}}\right) r+1\right]
$$

If the quadratic term (in square brackets) has no real zeros (or a double zero at $r=1)$ then $u^{\prime}(r)$ is always positive for $0<r<1$. Hence $u(r)<1$ for all $r$ in $r_{i}<r<1$. If the quadratic term has two distinct zeros, then 1 lies between them and $u^{\prime}(1)<0$. In this case there would have to be a range $r_{i}<r<r_{i}^{\prime}$ in which $u(r)<1$ and a range $r_{i}^{\prime}<r<1$ in which $u(r)>1$.

It is easily seen that the term in square brackets has no real zeros or a double zero at $r=1$ if $r_{i} \geqq 2-3^{1 / 2}\left(r_{i}\right.$ is restricted to lie in the interval $\left.0 \leqq r_{i}<1\right)$. In this case the minimum of $V\left(\rho^{2}\right)$ occurs at $\rho^{2}=\left[R^{2}-\delta^{2}\right]^{1 / 2}$.

Suppose $r_{i}<2-3^{1 / 2}$. Then $u(r)>1$ in some interval $r_{i}^{\prime}<r<1$. Setting $u(r)=1$, we find that $r_{i}^{\prime}$ must be a root of

$$
r^{2}-\frac{\left(1-6 r_{i}^{2}+r_{i}^{4}\right)}{r_{i}\left(1+r_{i}^{2}\right)} r+1=0 .
$$

This quadratic expression has only one root less than 1 . We therefore have proved

LEMma 1. Let $z_{i} \in U$. Then

$$
m\left(r, r_{i}\right) \leqq \operatorname{Re}\left\{\frac{z \Psi^{\prime}\left(z, z_{i}\right)}{\Psi\left(z, z_{i}\right)}\right\} \leqq M\left(r, r_{i}\right)
$$

where

and

$$
\begin{array}{rlrl}
M\left(r, r_{i}\right) & =-\frac{r_{i}\left(1-r^{2}\right)}{\left(r+r_{i}\right)\left(1+r_{i} r\right)} & & \text { if } 0 \leqq r<r_{i} ; \\
& =\frac{r_{i}\left(1-r^{2}\right)}{\left(r-r_{i}\right)\left(1-r_{i} r\right)} & \text { if } r_{i}<r<1 ;
\end{array}
$$

$$
\begin{aligned}
m\left(r, r_{i}\right) & =-\frac{r_{i}\left(1-r^{2}\right)}{\left(r_{i}-r\right)\left(1-r_{i} r\right)} \quad \text { if } 0 \leqq r<r_{i} \\
& =-\frac{\left[\left(1-r_{i}^{2} r^{2}\right)^{1 / 2}-\left(r^{2}-r_{i}^{2}\right)^{1 / 2}\right]^{2}}{2\left(1-r^{2}\right)\left(1-r_{i}^{2}\right)}
\end{aligned}
$$


if $r_{i}<r<1$ and $r_{i} \geqq 2-3^{1 / 2}$ or $r_{i}<r<r_{i}^{\prime}$ and $r_{i}<2-3^{1 / 2}$;

$$
=-\frac{r_{i}\left(1-r^{2}\right)}{\left(r+r_{i}\right)\left(1+r_{i} r\right)} \quad \text { if } r_{i}^{\prime} \leqq r<1 \text { and } r_{i}<2-3^{1 / 2} \text {. }
$$

Here, $r_{i}^{\prime}$ is the root of

$$
r^{2}-\frac{\left(1-6 r_{i}^{2}+r_{i}^{4}\right)}{r_{i}\left(1+r_{i}^{2}\right)} r+1=0
$$

which is between 0 and 1 . These bounds are all sharp. If $r_{i}<r<1$, then for any $r_{i}$

$$
m\left(r, r_{i}\right) \geqq-\frac{\left[\left(1-r_{i}^{2} r^{2}\right)^{1 / 2}-\left(r^{2}-r_{i}^{2}\right)^{1 / 2}\right]^{2}}{2\left(1-r^{2}\right)\left(1-r_{i}^{2}\right)}
$$

The last remark in the lemma follows from the fact that $V\left(\rho^{2}\right)$ has its minimum at $\rho^{2}=\left[R^{2}-\delta^{2}\right]^{1 / 2}$ even if this is larger than $(R+\delta)^{2}$. (Observe that $m\left(r, r_{i}\right)=M\left(r, r_{i}\right)$ $=0$ if $r_{i}=0$.)

This lemma can be used to put bounds on $\operatorname{Re}\left\{z f^{\prime}(z) / f(z)\right\}$ for any $f \in W_{p}$. Using the known bounds for starlike univalent functions, we have

$$
p\left(\frac{1-r}{1+r}\right)+\sum_{i=1}^{p} m\left(r, r_{i}\right) \leqq \operatorname{Re}\left\{\frac{z f^{\prime}(z)}{f(z)}\right\} \leqq p\left(\frac{1+r}{1-r}\right)+\sum_{i=1}^{p} M\left(r, r_{i}\right) .
$$

(The paper of Bender [1] contains part of this result.) However, it is much more interesting to attempt to find bounds which depend only on $R_{m}$ and $R_{M}$, or are independent of the $r_{i}$ entirely.

THEOREM 4. Let $f(z) \in W_{p}$, and let $M(r)$ and $m(r)$ be the supremum and infimum respectively of $\operatorname{Re}\left\{z f^{\prime}(z) \mid f(z)\right\}$ on $|z|=r$. Then

$$
\begin{aligned}
M(r) & \leqq \frac{p}{2} \frac{(1+r)^{3}}{(1-r)\left(1+r^{2}\right)} \quad \text { if } 0 \leqq r<R_{m} \\
& \leqq p\left(\frac{1+r}{1-r}\right) \frac{\left(1-R_{M}\right)^{2} r}{\left(r-R_{M}\right)\left(1-R_{M} r\right)} \quad \text { if } R_{M}<r<1
\end{aligned}
$$

and

$$
\begin{aligned}
m(r) & \geqq-p\left(\frac{1-r}{1+r}\right) \frac{\left(1+R_{m}\right)^{2} r}{\left(R_{m}-r\right)\left(1-R_{m} r\right)} \quad \text { if } 0 \leqq r<R_{m} ; \\
& \geqq p\left(\frac{1-r}{1+r}\right) \frac{\left(1-R_{M}\right)^{2} r}{\left(r+R_{M}\right)\left(1+R_{M} r\right)}, \\
& >p\left(\frac{1-r}{1+r}\right) \frac{2 \beta r}{(r+\beta)(1+\beta r)},
\end{aligned}
$$

if $r^{\prime}<r<1$, all $r_{i}<2-3^{1 / 2}, \beta=2-3^{1 / 2}$. 
In this last formula, $r^{\prime}=\max \left\{r_{i}^{\prime}\right\}$, where the $r_{i}^{\prime}$ are the roots of (19) which are between 0 and 1. Finally,

$$
m(r) \geqq \frac{p}{2} \frac{\left(1-4 r+r^{2}\right)}{(1-r)^{2}}+p \frac{\left(1-R_{M}^{2} r^{2}\right)^{1 / 2}\left(r^{2}-R_{M}^{2}\right)^{1 / 2}}{\left(1-r^{2}\right)\left(1-R_{M}^{2}\right)}, \quad R_{M}<r<1 .
$$

Inequalities (23), (24), and (25) are sharp. Inequalities (22) and (26) cannot be improved upon without specifying the $r_{i}$ more closely. Inequality (27) is sharp if all $r_{i}=R_{M} \geqq 2-3^{1 / 2}$.

Proof. We start with the proof of (22). If $0 \leqq r<R_{m}$, then for each $i$,

$$
\frac{1}{M\left(r, r_{i}\right)}=-\frac{1}{\left(1-r^{2}\right)}\left[1+r^{2}+r\left(\frac{1}{r_{i}}+r_{i}\right)\right] \text {. }
$$

For a fixed $r$, this is an increasing function of $r_{i}$ for $r \leqq r_{i}<1$. Its minimum occurs when $r_{i}=r$. Thus, $M\left(r, r_{i}\right)<M(r, r)=-\frac{1}{2}\left(1-r^{2}\right) /\left(1+r^{2}\right)$. Putting this into the right hand side of (21) gives (22).

In exactly the same way we see that $M\left(r, r_{i}\right)$ is an increasing function of $r_{i}$ for a fixed $r$ and $0 \leqq r_{i}<r$. Since it becomes infinite as $r_{i} \rightarrow r$, our best bound is obtained when $r_{i}=R_{M}$. Putting this into (21) gives (23). This result is sharp, being attained when all $z_{i}=R_{M}, h(z)=z /(1-z)^{2}$ and $z=r$.

In looking at the minimum, we see that

$$
\frac{1}{m\left(r, r_{i}\right)}=\frac{1}{\left(1-r^{2}\right)}\left[-1-r^{2}+r\left(\frac{1}{r_{i}}+r_{i}\right)\right]
$$

if $r<r_{i}<1$. It is then easy to see that $m\left(r, r_{i}\right)$ is negative and monotone increasing as a function of $r_{i}$ in this range. Its minimum is therefore taken on when $r_{i}=R_{m}$. (24) then follows easily. This too is sharp, being achieved when all $z_{i}=R_{m}, z=r$ and $h=z /(1+z)^{2}$.

Next, if $r_{i}<\beta=2-3^{1 / 2}$ and $r>r_{i}^{\prime}$, then $m\left(r, r_{i}\right)$, given by (18) is of the same form as $M\left(r, r_{i}\right)$ given by (14). Therefore $m\left(r, r_{i}\right)$ is decreasing as a function of $r_{i}$, while $r$ is held fixed. This gives

$$
m\left(r, r_{i}\right) \geqq m\left(r, R_{M}\right)>m(r, \beta) .
$$

Evaluating these, and putting them into (21) gives (25) and (26). It is easily seen that (25) is sharp and (26) cannot be improved. Observe that as $r_{i} \rightarrow \beta, r_{i}^{\prime} \rightarrow 1$, so (26) can never actually be obtained.

The final bound is obtained from (17) and (20). A straightforward computation gives

$$
\begin{aligned}
m\left(r, r_{i}\right) & =-\frac{\left(1+r^{2}\right)}{2\left(1-r^{2}\right)}+\frac{\left(1-r_{i}^{2} r^{2}\right)^{1 / 2}\left(r^{2}-r_{i}^{2}\right)^{1 / 2}}{\left(1-r^{2}\right)\left(1-r_{i}^{2}\right)} \\
& =-\frac{\left(1+r^{2}\right)}{2\left(1-r^{2}\right)}+\left[\frac{r^{2}}{\left(1-r^{2}\right)^{2}}+\frac{1}{\left(1-r_{i}^{2}\right)}-\frac{1}{\left(1-r_{i}^{2}\right)^{2}}\right]^{1 / 2}
\end{aligned}
$$


This is easily shown to be an increasing function of $\left(1-r_{i}^{2}\right)$ and hence a decreasing function of $r_{i}$. The minimum will be attained at the largest possible value of $r_{i}$, i.e. $r_{i}=R_{M}$. Putting this in, gives (27).

If all $z_{i}=R_{M} \geqq \beta=2-3^{1 / 2}$, then the minimum of $\operatorname{Re}\left\{z \Psi^{\prime} / \Psi(z, R)\right\}$ is actually achieved at some point $z=r e^{i \tau}$ where $\tau$ depends on $r$ and $R_{M}$. Thus, (27) is sharp, the minimum occurring for $h=z /\left(1+e^{-i \tau} z\right)^{2}$. It is interesting to notice that a different function is required for each $r$. That is, (27) is not simultaneously sharp for all $r>R_{M}$.

From (26) we easily prove

CoROllary 1. Let $f(z) \in W_{p}$ and suppose that all of the zeros of $f(z)$ are in the disc $\left\{z|| z \mid<2-3^{1 / 2}\right\}$. Then there exists some annulus $A=\left\{z\left|r^{\prime}<\right| z \mid<1\right\}$ such that

$$
\operatorname{Re}\left\{z f^{\prime}(z) / f(z)\right\}>0
$$

for all $z \in A$.

It is this property that has been used to define $p$-valent starlike functions in most earlier works ([2], [4] for example).

Finally, we end by giving a useful estimate for the asymptotic behavior of the lower bound of $\operatorname{Re}\left\{z f^{\prime}(z) \mid f(z)\right\}$ near $|z|=1$. This is obtained by making a Taylor's series expansion of (27).

Corollary 2. Let $f(z) \in W_{p}$. Set $t=1-|z|$. Then if $|z|>R_{M}$

$$
\operatorname{Re}\left\{z f^{\prime}(z) \mid f(z)\right\} \geqq u(t)
$$

where

$$
u(t)=\frac{p\left(1-6 R_{M}^{2}+R_{M}^{4}\right)}{4\left(1-R_{M}^{2}\right)^{2}}\left(t+\frac{1}{2} t^{2}\right)+o\left(t^{2}\right)
$$

Notice that if $R_{M}>2-3^{1 / 2}$, the lead coefficient of this expansion is negative.

\section{REFERENCES}

1. John Bender, Some extremal theorems for multivalently star-like functions, Duke Math. J. 29 (1962), 101-106.

2. A. W. Goodman, On the Schwarz-Christoffel transformation and p-valent functions, Trans. Amer. Math. Soc. 68 (1950), 204-223.

3. J. A. Hummel, Multivalent starlike functions, J. Analyse Math. 18 (1967), 133-160.

4. M. S. Robertson, Multivalently star-like functions, Duke Math. J. 20 (1953), 539-549.

\section{IMPERIAL COLLEGE,}

UNIVERSITY OF LONDON

UNIVERSITY OF MARYLAND,

College Park, Maryland 